\title{
Does Syncope Predict Mortality in Patients With Acute Pulmonary Embolism? A Retrospective Review
}

\author{
Umair Iqbal $^{\mathrm{a}, \mathrm{c}}$, Ayesha Jameel ${ }^{\mathrm{a}}$, Hafsa Anwarb ${ }^{\mathrm{b}}$, Melissa B. Scribani ${ }^{\mathrm{a}}$, \\ Edward Bischof ${ }^{\mathrm{a}}$, Ahmad Chaudhary ${ }^{\mathrm{a}}$
}

\begin{abstract}
Background: Acute pulmonary embolism (APE) is a potentially fatal disease with high mortality. Prior studies have shown an increased frequency of central localization of the clot, right ventricular dysfunction and elevated troponin in patients who present with syncope and APE. Existing evidence regarding mortality and length of hospital stay in these patients is unclear.

Methods: We retrospectively reviewed electronic medical records of patients who were admitted in a tertiary care hospital in rural Upstate New York and diagnosed with APE from July 2014 to July 2016. Two hundred nineteen patients were reviewed in two groups: patients who presented with syncope and those without syncope.
\end{abstract}

Results: The prevalence of syncope was found to be $6.8 \%(15 / 219)$. Hypotension on admission was more common among patients with syncope compared to no syncope $(26.7 \%$ and $7.4 \%$, respectively, $\mathrm{P}=$ 0.03 ). A clinically significant difference was found in 30-day mortality among those with syncope versus no syncope $(21.3 \%$ vs. $7.4 \%$, P $=0.096$ ). No significant difference was found in length of stay (mean 6.7 days in patients with syncope vs. 6.4 without syncope, $\mathrm{P}=0.783$ ), central localization $(26.7 \%$ with syncope vs. $43.2 \%$ without syncope, $\mathrm{P}=0.21)$ or troponin elevation $(46.2 \%$ in patients with syncope vs. $27.9 \%$ without syncope, $\mathrm{P}=0.205)$. On multivariable analysis, hypotension was significantly higher among those with syncope (odds ratio: $5.23, \mathrm{P}=0.0148$ )

Conclusion: This study suggests 30-day mortality may be higher among patients with syncope. It is important to risk stratify patients on admission in order to reduce mortality and morbidity associated with lethal disease.

Keywords: Acute pulmonary embolism; Syncope; Right ventricular dysfunction; Central pulmonary embolism

Manuscript accepted for publication April 10, 2017

aBassett Medical Center, One Atwell Road, Cooperstown, NY 13326, USA bDow University of Health and Sciences, Karachi, Pakistan

${ }^{\mathrm{c} C}$ Corresponding Author: Umair Iqbal, Bassett Medical Center, One Atwell Road, Cooperstown, NY 13326, USA. Email: umair.iqbal@bassett.org

doi: https://doi.org/10.14740/jocmr3037w

\section{Introduction}

Acute pulmonary embolism (APE) is a potentially fatal disease with high mortality. Incidence of pulmonary embolism is around 100 - 200 cases per 100,000 [1]. Overall incidence is higher in males and it increases with advancing age, with some studies showing incidence to be $>500$ per 100,000 after the age of 75 years $[2,3]$. Pulmonary embolism has a high mortality with recent studies showing 30 -day mortality to be $4 \%$ and 90-day mortality $13 \%$ [4]. Again the mortality is higher with advancing age $[2,3]$. The typical symptoms of APE include chest pain, dyspnea and hemoptysis.

Syncope is an uncommon presentation of APE with prevalence around 10\% [5-9]. Data regarding mortality and length of stay in this subset of patients with APE are controversial, with some studies suggesting increased mortality $[6,10]$, while other suggested no difference in mortality $[11,12]$. One study also reported syncope to be associated with an improved prognosis [13]. We did this retrospective review to clarify the association of syncope in pulmonary embolism patients with mortality.

\section{Patients and Methods}

We retrospectively reviewed electronic medical records of patients who were admitted in a tertiary care hospital in rural Upstate New York and diagnosed with APE from July 2014 to July 2016. All patients over 18 years of age admitted with diagnosis of APE (ICD 10 codes: I26.0, I26.9) were included in the study. Patients who developed APE during hospitalization were excluded from the study.

Syncope was defined as transient loss of consciousness ( $<$ $1 \mathrm{~min}$ ) with spontaneous resolution. APE was diagnosed on the basis of CT scan, high probability V/Q scan or venous Doppler ultrasound with associated symptoms. Two hundred nineteen patients met the inclusion criteria and were reviewed in two groups: patients who presented with syncope and those without syncope. Four of 219 were diagnosed with high probability $\mathrm{V} / \mathrm{Q}$ scan, two patients were diagnosed with venous duplex and 213 were diagnosed with CT scan on chest.

We defined right ventricular dysfunction (RVD) as right ventricular (RV)/left ventricular (LV) ratio $>0.9$ or RV hypokinesis on echocardiogram as defined by the American Heart 
Table 1. Results From Univariate Tests (Chi-Square/Fisher's Exact Tests for Categorical Variables, $t$-Tests for Continuous Variables)

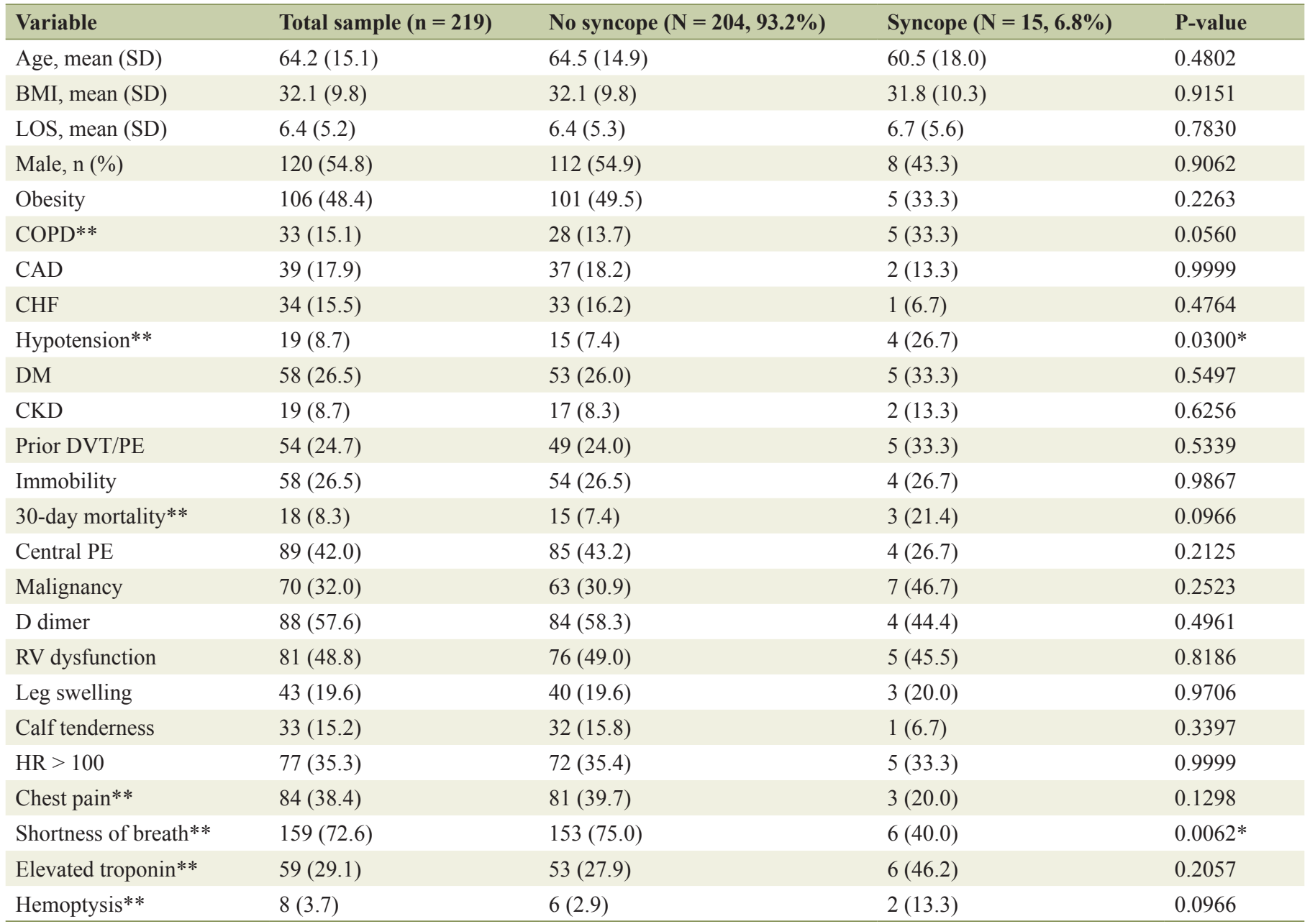

*Statistically significant. **Variables included in stepwise multivariable logistic regression (P-value less than 0.20 on univariate tests).

Association or RV dilation on CT scan. Location of thrombus was classified as central if it is located in one of the main pulmonary arteries or peripheral if involving segmental, subsegmental or lobar arteries.

\section{Statistical analysis}

To identify univariate associations between syncope and subject characteristics, the Chi-square/Fisher's exact test and $t$ tests were used. $\mathrm{P}$ value of $<0.05$ was considered statistically significant. All variables with $\mathrm{P}<0.2$ in the initial analysis were included in a stepwise multivariable logistic regression model to identify independent predictors of syncope. All analyses were carried out using SAS 9.3 (Cary, NC, USA).

\section{Results}

The prevalence of syncope was found to be $6.8 \%(15 / 219)$ (Table 1). Central localization of the clot was present in $42 \%$ of patients (89/219). Shortness of breath was the most common presenting symptom present in $72.6 \%$ followed by chest pain in $38.2 \%$. On physical exam, calf tenderness and tachycardia (heart rate $(\mathrm{HR})>100)$ were common. Troponin elevation defined as troponin $>0.05$ was present in $29 \%$ of patients.

The overall 30-day mortality associated with APE was $8.3 \%$ (18/219). The 30-day mortality was higher among those with syncope versus no syncope $(21.3 \%$ vs. $7.4 \%, \mathrm{P}=0.096)$, though the result was not statistically significant. No statistically significant difference was found in length of stay (mean 6.7 days in patients with syncope vs. 6.4 without syncope, $\mathrm{P}$ $=0.783)$, central localization $(26.7 \%$ with syncope vs. $43.2 \%$ without syncope, $\mathrm{P}=0.21)$ or troponin elevation $(46.2 \%$ in patients with syncope vs. $27.9 \%$ without syncope, $\mathrm{P}=0.205)$.

Hypotension on admission was more common among patients with syncope compared to no syncope $(26.7 \%$ and $7.4 \%$, respectively, $\mathrm{P}=0.03)$. Chronic obstructive pulmonary disease (COPD) was more common in patients with syncope $(33.3 \%$ with syncope vs. $13.7 \%$ without syncope, $\mathrm{P}=0.056)$, while shortness of breath is more common in patients who did not present with syncope $(40 \%$ with syncope and $75 \%$ without 
Table 2. Results of Logistic Regression

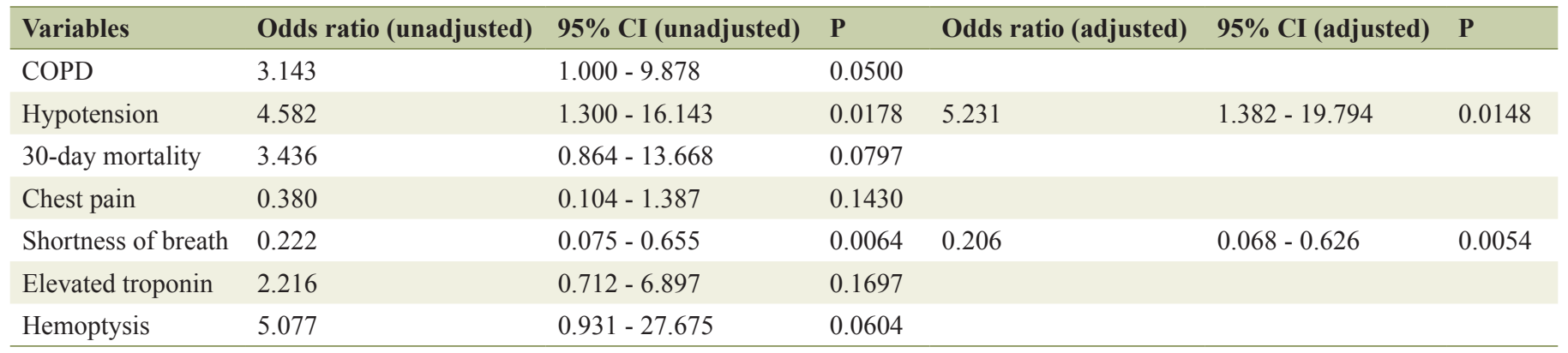

Hypotension and shortness of breath were retained as independent predictors of syncope after stepwise selection.

syncope, $\mathrm{P}=0.0062$ ). No difference was found in other comorbidities in the two groups including coronary artery disease (CAD), diabetes, chronic kidney disease (CKD) and congestive heart failure (CHF).

All variables with $\mathrm{P}<0.2$ were included in a stepwise multivariable logistic regression model to predict syncope. Only hypotension and shortness of breath were retained as independent predictors of syncope after stepwise selection (Table 2). Hypotension was significantly higher among those with syncope (odds ratio (OR): $5.23 ; \mathrm{P}=0.0148$ ) and shortness of breath was higher in patients without syncope (OR: 0.206; P $=0.0054)$.

\section{Discussion}

APE is directly responsible for 100,000 deaths annually in United States [14]. The classic presentation of PE includes chest pain, dyspnea and hemoptysis, but it can present atypically as abdominal pain, seizure and cardiac arrhythmias, most commonly new onset atrial fibrillation [15]. Syncope is an atypical presentation of APE and can be present in $10 \%$ of the patients. The prevalence of syncope in our study was $6.8 \%$ which is slightly lower than what is reported elsewhere.

A review of the literature describes three possible mechanisms of syncope in APE. First, APE can cause sudden right heart failure which decreases left ventricular filling and ultimately result in low cardiac output, hypotension and decreased cerebral perfusion. Second, PE can cause cardiac arrhythmias which can result in a syncopal episode. Lastly, blockage of the pulmonary artery by embolus can stimulate ventricular mechanoreceptors which increase the vagal response causing vagalinduced syncope $[5,16]$.

Syncope is generally considered a poor prognostic factor in APE. Multiple studies have shown syncope in APE to be associated with higher mortality $[6,10]$. A study showed syncope to be associated with high inpatient mortality with odds of 3 [17]. In the International Cooperative Pulmonary Embolism Registry, the 3-month mortality rate of patients with syncope was $26.8 \%$, whereas the overall mortality rate was $17 \%$ [18]. Although mortality was higher in patients with syncope in our study, this was not statistically significant. These results may be clinically significant.

Several studies in the past showed syncope in patients with APE to be associated with increased prevalence of central emboli, RVD, and troponin positivity [5, 16]. Our study did not show any such association of these variables with syncope. Several factors can be contributed to these results. Several criteria have been described in the literature for the diagnosis of RVD in the echocardiogram or RV dilation in the CT scan. We only included patients as per AHA definition of RVD as described above. This may underscore the patients diagnosed with RVD in our study unlike other studies. It has been postulated that central pulmonary embolism can cause sudden RV strain as described above and cause decreased cerebral perfusion causing syncope. Our study results may support vasovagal reflex as the likely cause of syncope. Our study did show an increased association of hypotension and syncope in APE. The results are similar to studies done in the past which also showed syncope to be associated with hemodynamic instability [16]. The likely mechanism of this hypotension is similar to what is described above.

A recent study done on patients admitted with first episode of syncope showed every one in six patients have a diagnosis of APE [19]. These numbers are really striking and clinicians should consider the diagnosis of APE in every patient with syncope by calculating the probability through Wells score [20]. Given that syncope may be relatively common in patients admitted to hospital, it is essential to recognize the potential increased mortality in this group as demonstrated in our study. Further studies are warranted to evaluate this association of higher mortality in patient with APE presenting as syncope.

\section{Limitation}

The design of our study is retrospective. Patient population in the syncope group is small. This may limit the interpretation of our results. A larger study may help to further elucidate the association between syncope and 30-day mortality, as well as other variables such as elevated troponin and hemoptysis.

\section{Conclusion}

This study suggests 30-day mortality may be higher among patients with syncope. It is important to determine potential risk factors for morbidity and mortality in APE since it may 
affect management and, thus, further study of the association of syncope and mortality in APE may be warranted.

\section{Acknowledgments}

Jennifer Victory, RN, Bassett Medical Center Research Department.

\section{References}

1. Belohlavek J, Dytrych V, Linhart A. Pulmonary embolism, part I: Epidemiology, risk factors and risk stratification, pathophysiology, clinical presentation, diagnosis and nonthrombotic pulmonary embolism. Exp Clin Cardiol. 2013;18(2):129-138.

2. Tagalakis V, Patenaude V, Kahn SR, Suissa S. Incidence of and mortality from venous thromboembolism in a realworld population: the Q-VTE Study Cohort. Am J Med. 2013;126(9):832 e813-821.

3. Silverstein MD, Heit JA, Mohr DN, Petterson TM, O'Fallon WM, Melton LJ, 3rd. Trends in the incidence of deep vein thrombosis and pulmonary embolism: a 25-year population-based study. Arch Intern Med. 1998;158(6):585-593.

4. Alotaibi GS, Wu C, Senthilselvan A, McMurtry MS. Secular Trends in Incidence and Mortality of Acute Venous Thromboembolism: The AB-VTE Population-Based Study. Am J Med. 2016;129(8):879 e819-825.

5. Altinsoy B, Erboy F, Tanriverdi H, Uygur F, Ornek T, Atalay F, Tor M. Syncope as a presentation of acute pulmonary embolism. Ther Clin Risk Manag. 2016;12:10231028.

6. Thames MD, Alpert JS, Dalen JE. Syncope in patients with pulmonary embolism. JAMA. 1977;238(23):25092511.

7. Ferrari E, Baudouy M, Cerboni P, Tibi T, Guigner A, Leonetti J, Bory M, et al. Clinical epidemiology of venous thromboembolic disease. Results of a French Multicentre Registry. Eur Heart J. 1997;18(4):685-691.

8. Calvo-Romero JM, Perez-Miranda M, Bureo-Dacal P. Syncope in acute pulmonary embolism. Eur J Emerg Med. 2004;11(4):208-209.

9. Morpurgo M, Zonzin P. Syncope in acute pulmonary embolism. Ital Heart J. 2004;5(1):3-5.

10. Koutkia P, Wachtel TJ. Pulmonary embolism presenting as syncope: case report and review of the literature. Heart Lung. 1999;28(5):342-347.

11. Jenab Y, Lotfi-Tokaldany M, Alemzadeh-Ansari MJ, Seyyedi SR, Shirani S, Soudaee M, Ghaffari-Marandi N. Correlates of syncope in patients with acute pulmonary thromboembolism. Clin Appl Thromb Hemost. 2015;21(8):772-776.

12. Jimenez D, Diaz G, Valle M, Marti D, Escobar C, Vidal $\mathrm{R}$, Picher J, et al. [Prognostic value of syncope in the presentation of pulmonary embolism]. Arch Bronconeumol. 2005;41(7):385-388.

13. Seyyedi SR, Jenab Y, Tokaldany ML, Shirani S, Sadeghian S, Jalali A. Syncope paradox in the outcome of patients with pulmonary thromboembolism: short-term and midterm outcome. Clin Respir J. 2016;10(1):90-97.

14. Horlander KT, Mannino DM, Leeper KV. Pulmonary embolism mortality in the United States, 1979-1998: an analysis using multiple-cause mortality data. Arch Intern Med. 2003;163(14):1711-1717.

15. Stein PD, Terrin ML, Hales CA, Palevsky HI, Saltzman HA, Thompson BT, Weg JG. Clinical, laboratory, roentgenographic, and electrocardiographic findings in patients with acute pulmonary embolism and no pre-existing cardiac or pulmonary disease. Chest. 1991;100(3):598-603.

16. Castelli R, Tarsia P, Tantardini C, Pantaleo G, Guariglia A, Porro F. Syncope in patients with pulmonary embolism: comparison between patients with syncope as the presenting symptom of pulmonary embolism and patients with pulmonary embolism without syncope. Vasc Med. 2003;8(4):257-261.

17. Kumasaka N, Sakuma M, Shirato K. Clinical features and predictors of in-hospital mortality in patients with acute and chronic pulmonary thromboembolism. Intern Med. 2000;39(12):1038-1043.

18. Goldhaber SZ, Visani L, De Rosa M. Acute pulmonary embolism: clinical outcomes in the International Cooperative Pulmonary Embolism Registry (ICOPER). Lancet. 1999;353(9162):1386-1389.

19. Prandoni P, Lensing AW, Prins MH, Ciammaichella M, Perlati M, Mumoli N, Bucherini E, et al. Prevalence of Pulmonary Embolism among Patients Hospitalized for Syncope. N Engl J Med. 2016;375(16):1524-1531.

20. Wells PS, Anderson DR, Rodger M, Ginsberg JS, Kearon C, Gent M, Turpie AG, et al. Derivation of a simple clinical model to categorize patients probability of pulmonary embolism: increasing the models utility with the SimpliRED D-dimer. Thromb Haemost. 2000;83(3):416-420. 